\title{
Precise determination of the lattice spacing in full lattice QCD
}

\author{
C. T. H. Davies,, , F E. Follana, ${ }^{2}$ I. D. Kendall, ${ }^{1}$ G. Peter Lepage, ${ }^{3}$ and C. McNeile ${ }^{1}$ \\ (HPQCD collaboration), , † \\ ${ }^{1}$ Department of Physics and Astronomy, University of Glasgow, Glasgow, G12 8QQ, UK \\ ${ }^{2}$ Department of Theoretical Physics, University of Zaragoza, E-50009 Zaragoza, Spain \\ ${ }^{3}$ Laboratory of Elementary-Particle Physics, Cornell University, Ithaca, New York 14853, USA
}

(Dated: October 29, 2018)

\begin{abstract}
We compare three different methods to determine the lattice spacing in lattice QCD and give results from calculations on the MILC ensembles of configurations that include the effect of $u, d$ and $s$ sea quarks. It is useful, for ensemble to ensemble comparison, to express the results as giving a physical value for $r_{1}$, a parameter from the heavy quark potential. Combining the three methods gives a value for $r_{1}$ in the continuum limit of $0.3133(23)(3) \mathrm{fm}$. Using the MILC values for $r_{0} / r_{1}$, this corresponds to a value for the $r_{0}$ parameter of $0.4661(38) \mathrm{fm}$. We also discuss how to use the $\eta_{s}$ for determining the lattice spacing and tuning the $s$-quark mass accurately, by giving values for $m_{\eta_{s}}(0.6858(40) \mathrm{GeV})$ and $f_{\eta_{s}}(0.1815(10) \mathrm{GeV})$.
\end{abstract}

\section{INTRODUCTION}

Results from lattice QCD calculations are generally computed in units of the lattice spacing $a$ used in the simulation. The lattice spacing must be computed separately and divided out in order to convert these results into physical units $(\mathrm{GeV}, \mathrm{fm} . .$.$) , for comparison with$ experiment. Any error in the lattice spacing determination feeds into most other quantities from lattice QCD, and, in many cases, it is among the dominant sources of errors. For example, in our determination of the decay constant of the $D_{s}$ meson [1, $1 \%$ of the total error of $1.3 \%$ comes from the $1.5 \%$ uncertainty in the value of the lattice spacing. Reducing the error on the lattice spacing is then very important for increasing the precision of the realistic lattice QCD calculations now possible [2].

Generally the value of the lattice spacing is determined by comparing values from the simulation, in lattice units, with values from experiment, in physical units. A lattice simulation, for example, might give a value for the pion decay constant in lattice units: $a f_{\pi}^{\text {lat }}$. Dividing by the experimental value $f_{\pi}^{\exp }$ in $\mathrm{GeV}$ gives a value for the lattice spacing, $a=\left(a f_{\pi}^{\text {lat }}\right) / f_{\pi}^{\exp }$, in inverse $\mathrm{GeV}$. This lattice spacing can then be used to convert other simulation results from lattice units to physical units.

Lattice spacings determined in this way are inherently ambiguous because lattice simulations are never exact. In particular the use of a nonzero lattice spacing causes lattice quantites, like $f_{\pi}^{\text {lat }}$, to deviate from their physical values, in this case $f_{\pi}^{\text {exp }}$. Such errors differ from quantity to quantity, and therefore so will values for the lattice spacing that are computed from these quantities. Such differences, however, vanish in the continuum limit, $a \rightarrow$ 0 , and so do not affect lattice predictions that have been extrapolated to $a=0$.

* c.davies@physics.gla.ac.uk

tURL: http://wWw.physics.gla.ac.uk/HPQCD
In principle, any dimensionful quantity can be used to determine the lattice spacing, but some quantities are more useful than others. Ideally one wants quantities that are easily computed, free of other types of simulation error, largely independent of lattice parameters other than the lattice spacing, and well measured in experiments. Use of the pion decay constant, for example, is not ideal. This decay constant is quite sensitive to the $u$ and $d$ quark masses, which are generally too large in current simulations; accurate values for the decay constant can be obtained only after chiral extrapolations of the simulation data to the physical quark masses. This greatly complicates the use of the decay constant to set the lattice spacing.

One physical quantity that is very easy to calculate in lattice simulations is the $r_{1}$ parameter derived from the potential $V(r)$ between two infinite-mass quarks separated by distance $r$. Parameter $r_{1}$ is defined implicitly by the equation $r_{1}^{2} F\left(r_{1}\right)=C$ where $F(r) \equiv d V / d r$ and $C=1$ 3. (Taking $C=1.65$ gives the original such standard parameter, $r_{0}$ [4.) This quantity is easily calculated, in lattice units (that is, $r_{1} / a$ ), to better than $1 \%$. Unlike the pion decay constant, it is only weakly dependent upon the quark masses. It would be an ideal choice for setting the lattice spacing except for the fact that there is no experimental value for the physical $r_{1}$ - this must be estimated instead from other lattice calculations.

In this paper we examine three other quantities that can be used to determine the lattice spacing: 1) the radial excitation energy in the $\Upsilon$ system $\left(m_{\Upsilon^{\prime}}-m_{\Upsilon}\right)$; 2 ) the mass difference between the $D_{s}$ meson and one half the $\eta_{c}$ mass; and 3) the decay constant of the fictitious $\eta_{s}$ particle, which can be related accurately to $f_{K}$ and $f_{\pi}$. The valence-quark masses are easily tuned in each case and each quantity is relatively insensitive to sea-quark masses. Consequently each of these quantities can be used to generate lattice spacings on an ensembleby-ensemble basis.

None of these quantities can be computed as accurately as $r_{1} / a$ in simulations, but we can combine simulation re- 
sults for them with values for $r_{1} / a$ to obtain very accurate estimates for the physical value of $r_{1}$. Given $r_{1}$, the different values of $r_{1} / a$ can be used to obtain accurate lattice spacings for each of the simulations we discuss here and any other simulations where $r_{1} / a$ has been computed.

Of our three quantities, the $\eta_{s}$ decay constant gives the most accurate results. The $\eta_{s}$ is a fictitious meson, however, and so its "experimental" properties must be related to those of real mesons using simulations. The $\eta_{s}$ is particularly closely related to the $\pi$ and $K$ mesons. As we will show, its mass and decay constant can be accurately related to those of the $\pi$ and $K$ through a chiral analysis of simulation data for a variety of quark masses and lattice spacings. Such an analysis also gives an independent, fourth estimate of $r_{1}$.

We describe in section 2 the three primary methods we have used to obtain lattice spacings for a wide variety of simulations. Each can be used to generate an estimate for the physical value of $r_{1}$, given values of $r_{1} / a$. In section 3 we combine the three analyses to generate a single, combined estimate for $r_{1}$. This can then be used to covert the $r_{1} / a$ values into a determination of $a$ on each ensemble. We also demonstrate how to determine the lattice spacing from the $\eta_{s}$ without using $r_{1}$. The two methods are compared and shown to agree in the $a \rightarrow 0$ limit. In Section 4 we give a value for $r_{0}$ derived from our value of $r_{1}$ for comparison to others using that parameter. In section 5 summarize our results. Finally, we discuss the chiral analysis of decay constants and masses for the $\pi, K$ and their relation with those of the $\eta_{s}$ meson in Appendices A, B and C.

\section{LATTICE CALCULATION}

In Table I we list the parameter sets for the different MILC ensembles of gluon configurations that we have used here, although not all ensembles were used in every lattice spacing determination.

Values for the static-quark potential parameter $r_{1} / a$, in lattice units, were determined by the MILC collaboration [5]. They calculated the heavy quark potential by fitting Wilson loops of fixed spatial size as a function of lattice time. On the finest two sets of ensembles smeared time links were used to reduce statistical noise and a twostate exponential fit in time reduced the contamination from excited potentials. The heavy quark potential obtained was then fit as a function of spatial separation over the range between $0.2 \mathrm{fm}$ and $0.7 \mathrm{fm}$ to a Cornell potential with the addition of corrections for lattice artifacts. The point at which the condition for $r_{1}$ held was then determined from this fit. The errors given are statistical errors only, since discretisation effects are taken care of in our continuum extrapolations.

In what follows we will combine these values for $r_{1} / a$ with estimates of the lattice spacing $a$ determined using three different physical quantities to obtain estimates for the physical value of $r_{1}$ (that is, at zero lattice spacing and with correct sea-quark masses).

$$
\text { A. } \quad m_{\Upsilon^{\prime}}-m_{\Upsilon}
$$

The calculation of the spectrum of mesons formed as bound states of bottom quarks and antiquarks has been an important test for lattice QCD. There are many radial and orbital excitations below threshold for strong decay and so many gold-plated states, well-characterised experimentally. The radial and orbital excitation energies are almost identical for charmonium and bottomonium when spin-averaged [6] and so rather insensitive to the heavy quark mass. Heavy-quark vacuum polarization effects are tiny and so can be safely neglected. This makes these systems very suitable for the determination of the lattice spacing [7] and was one of the key calculations demonstrating the importance of including the effect of $u, d$ and $s$ sea quarks [2].

Here we improve on the calculations in [7] which used results from MILC super-coarse, coarse and fine ensembles and compared ensembles with and without sea quarks. We study only ensembles including sea quarks but include also very coarse and superfine ensembles for a wider range of lattice spacing values.

We calculate $b$-quark propagators on the MILC gluon field configurations using lattice NonRelativistic QCD (NRQCD) which has been developed over many years to handle well the physics of heavy quark systems on the lattice 8 . It makes a virtue of the nonrelativistic nature of bottomonium bound states $\left(v_{b}^{2} \approx 0.1\right.$ for the $\Upsilon$ ) by discarding the rest mass energy in favour of accurately handling typical momentum and energy scales inside the bound states. NRQCD can be matched to full QCD order by order in $v_{b}^{2}$ and $\alpha_{s}$. We work through $\mathcal{O}\left(v_{b}^{4}\right)$ in the nonrelativistic expansion and apply discretisation improvements through $\mathcal{O}\left(a^{2}\right)$ to $v_{b}^{2}$ terms and to chromomagnetic and chromoelectric field-dependent terms at $v_{b}^{4}$ (so that terms which induce fine structure are completely improved to $\mathcal{O}\left(a^{4}\right)$ ). An analysis of remaining systematic errors is given in [7].

The NonRelativistic Hamiltonian that we use is given by [9]:

$$
\begin{aligned}
a H= & a H_{0}+a \delta H \\
a H_{0}= & -\frac{\Delta^{(2)}}{2 a M_{b}}, \\
a \delta H= & -c_{1} \frac{\left(\Delta^{(2)}\right)^{2}}{8\left(a M_{b}\right)^{3}}+c_{2} \frac{i g}{8\left(a M_{b}\right)^{2}}(\nabla \cdot \tilde{\mathbf{E}}-\tilde{\mathbf{E}} \cdot \nabla) \\
& -c_{3} \frac{g}{8\left(a M_{b}\right)^{2}} \sigma \cdot(\tilde{\nabla} \times \tilde{\mathbf{E}}-\tilde{\mathbf{E}} \times \tilde{\nabla}) \\
& -c_{4} \frac{g}{2 a M_{b}} \sigma \cdot \tilde{\mathbf{B}}+c_{5} \frac{a^{2} \Delta^{(4)}}{24 a M_{b}} \\
& -c_{6} \frac{a\left(\Delta^{(2)}\right)^{2}}{16 n\left(a M_{b}\right)^{2}} .
\end{aligned}
$$

This is implemented in calculating $b$ quark propagators 
TABLE I: Ensembles (sets) of MILC configurations with gauge coupling $\beta$, size $L^{3} \times T$ and sea mass parameters $m_{l}^{\text {asq }}$ and $m_{s}^{\text {asq }}$ used for this analysis. The sea ASQTAD quark masses $(l=u / d)$ are given in the MILC convention where $u_{0}$ is the plaquette tadpole parameter. The lattice spacing values in units of $r_{1}$ after 'smoothing' are given in the third column [5]. Sets 1 and 2 are 'very coarse'; sets 3, 4 and 5, 'coarse'; sets 6 and 7 'fine'; set 8 'superfine' and set 9 'ultrafine'.

\begin{tabular}{lllllll}
\hline \hline Set & $\beta$ & $r_{1} / a$ & $a u_{0} m_{l}^{\text {asq }}$ & $a u_{0} m_{s}^{\text {asq }}$ & $L / a$ & $T / a$ \\
\hline 1 & 6.572 & $2.152(5)$ & 0.0097 & 0.0484 & 16 & 48 \\
2 & 6.586 & $2.138(4)$ & 0.0194 & 0.0484 & 16 & 48 \\
\hline 3 & 6.76 & $2.647(3)$ & 0.005 & 0.05 & 24 & 64 \\
4 & 6.76 & $2.618(3)$ & 0.01 & 0.05 & 20 & 64 \\
5 & 6.79 & $2.644(3)$ & 0.02 & 0.05 & 20 & 64 \\
\hline 6 & 7.09 & $3.699(3)$ & 0.0062 & 0.031 & 28 & 96 \\
7 & 7.11 & $3.712(4)$ & 0.0124 & 0.031 & 28 & 96 \\
\hline 8 & 7.46 & $5.296(7)$ & 0.0036 & 0.018 & 48 & 144 \\
\hline 9 & 7.81 & $7.115(20)$ & 0.0028 & 0.014 & 64 & 192 \\
\hline \hline
\end{tabular}

by evolving the $b$ quark Green's function on a single pass through the lattice using:

$$
\begin{aligned}
G(\vec{x}, t+1)= & \left(1-\frac{a \delta H}{2}\right)\left(1-\frac{a H_{0}}{2 n}\right)^{n} U_{t}^{\dagger}(x) \\
& \left(1-\frac{a H_{0}}{2 n}\right)^{n}\left(1-\frac{a \delta H}{2}\right) G(\vec{x}, t)
\end{aligned}
$$

with starting condition:

$$
G(\vec{x}, 0)=\phi(x) 1 .
$$

Here $\nabla$ is the symmetric lattice derivative and $\tilde{\nabla}$ is the improved derivative, $\tilde{\nabla}_{k}=\nabla_{k}-\nabla_{k}^{(3)} / 6 . \Delta^{(2)}$ is the standard lattice discretisation of the second derivative $\sum_{j} \nabla_{j}^{(2)}$ and $\Delta^{(4)}$ is $\sum_{j} \nabla_{j}^{(4)} . a M_{b}$ is the bare $b$ quark mass in lattice units.

$\phi(x)$ is a real spatial smearing function which multiplies a unit matrix in color and spin space as the starting point for the quark propagator. The antiquark propagator for a given source is then the complex conjugate of the Green's function obtained from eq. 2. When the quark and antiquark propagators are combined (with appropriate Pauli matrices for different $J^{P C}[10]$ ) into meson correlators, $\phi$ improves the overlap with particular ground and excited states for a better signal. This will be discussed further below.

$n$ is a stability parameter which is chosen to tame (unphysical) high momentum modes of the $b$ quark propagator which might otherwise cause the meson correlators to grow exponentially with time rather than fall. We used $n=4$ throughout this calculation instead of the value $n=2$ used earlier [7, so that we could work on finer lattices and keep the same value of $n$ for all ensembles. This means that discretisation errors are smoothly connected from one lattice spacing to another and the higher value of $n$ has the advantage of reducing some systematic errors.
TABLE II: Parameters used in our calculations of $b$ quark propagators and $b \bar{b}$ correlators on various MILC ensembles, numbered as in Table I] $M_{b} a$ is the bare $b$ quark mass in lattice units, $u_{O L}$ is the tadpole-improvement factor and the stability factor $n$ is taken as 4 everywhere. $n_{c f g}$ gives the number of gluon field configurations used from the ensemble and $n_{t}$ is the number of time sources for $b$ quark propagators per configuration. $T$ is the time length in lattice units of the propagators. $a_{0}$ is the size parameter for the quark smearing function $\phi_{e s}(x)$ given in eq. 4

\begin{tabular}{lllllll}
\hline \hline Set & $a M_{b}$ & $u_{0 L}$ & $n_{c f g}$ & $n_{t}$ & $T$ & $a_{0}$ \\
\hline 1 & 3.4 & 0.8218 & 631 & 24 & 32 & 0.83 \\
2 & 3.4 & 0.8225 & 631 & 24 & 32 & 0.83 \\
\hline 3 & 2.8 & 0.8362 & 2083 & 32 & 32 & 1.0 \\
4 & 2.8 & 0.8359 & 595 & 32 & 32 & 1.0 \\
\hline 6 & 1.95 & 0.8541 & 557 & 8 & 48 & 1.41 \\
\hline 8 & 1.34 & 0.8696 & 698 & 8 & 48 & 2.0 \\
\hline \hline
\end{tabular}

We use 'tadpole-improvement' [11 for all terms by dividing the gluon fields $U_{\mu}$ by a factor $u_{0}$ when they are read in. $u_{0}$ is taken as $u_{0 L}$, the value of the mean trace of the gluon field for that ensemble in lattice Landau gauge (where the trace is maximised). This removes, in a meanfield way, the disparity between lattice and continuum gluon fields induced by the fact that the lattice field is exponentially related to the continuum field. Single composite operators, such as $\Delta^{(4)}$, are expanded out fully so that all cancellations between $U$ and $U^{\dagger}$ are correctly tadpole-improved. This is not done for the $\left(1-a H_{0} / 2 n\right)^{n}$ terms. Table [II gives our parameters for the ensembles used in the $\Upsilon$ analysis.

In order to reduce statistical errors over our previous calculation we have investigated a number of improvements. The first was to look at different forms for the quark smearing $\phi(x)$. The simplest is a $\delta$ function but in addition we can take an arbitrary functional form for $\phi(x)$ provided that the gluon field configurations are gaugefixed, at least on a time-slice. The MILC configurations that we use here are fixed to Coulomb gauge. When a $b$ quark propagator from a $\delta$ source and a $\bar{b}$ propagator from a $\phi=f(x)$ source are combined a good overlap with a particular $\Upsilon$ state is expected when, in the language of a potential model, $f(x)$ is a good approximation to the wavefunction of that state. The ground state $\Upsilon(1 S)$ will dominate all $1^{--}$correlators eventually so that there is no advantage in including a smearing function that gives a good overlap with that state [12. Instead, to obtain a good signal for the $2 S-1 S$ splitting, we concentrated on functions that had very small overlap with the 1S state, and therefore had better information about radial excitations. A very good smearing for this was the function from [7] called $\phi_{e s}$ :

$$
\phi_{e s}(r)=\left(2 a_{0}-r\right) \exp \left(-r /\left(2 a_{0}\right)\right) .
$$

The size parameter, $a_{0}$, was tuned on coarse lattices to reduce the overlap of the correlator (known as the 'ee' correlator, see below) with the ground state, as judged 
by the small amplitude of the correlator at large times when the ground state dominates. $a_{0}$ was then scaled as appropriate to ensembles of different lattice spacing. Values are given in Table II. By combining $b$ and $\bar{b}$ propagators from $\delta$ function sources and $\phi_{e s}$ sources we are able to make up 3 different meson smearing functions: $l$ is from combining two $\delta$ sources; $e$ is from combining a $\phi_{e s}$ source with a $\delta$ source and $E$ is from combining two $\phi_{e s}$ sources (so that the composite meson smearing function is then the convolution of $\phi_{e s}$ with itself). $l, e$ and $E$ smearing functions can also be applied at the sink to make a $3 \times 3$ matrix of correlators, with notation $l l$, le, ee etc.

We also used a random wall source for our $b$ quark propagators, taking a set of $\mathrm{U}(1)$ random numbers, $r$, with unit norm at every point on a time slice, one set for each color of the $b$ quark propagator. These were combined with the smearing functions $\phi$ so that

$$
G(\vec{x}, 0)_{c_{1} c_{1}}=\sum_{\vec{y}} \phi(|\vec{x}-\vec{y}|) r\left(c_{1}, \vec{y}\right) 1_{\text {spin }}
$$

When quark and antiquark propagators are combined together the random noise cancels except where the initial spatial sites are the same and this effectively increases the number of meson correlators sampled. We find that the error on the ground state $\Upsilon$ energy is reduced by a factor of 3 on coarse lattices and 5 on fine lattices, when corrected to the same number of configurations. The excited state energy does not improve by the same factor, however. Indeed we found rather little improvement in the error on excited state energies which mirrors our experience with applying random wall sources to $B$ mesons [13. The inference is that random wall sources are much less effective in situations where the degradation of the signal/noise is exponential. We calculate propagators from many different time sources (which we then average over) per configuration to improve statistical precision further. The details of numbers of configurations and time sources are collected in Table II.

As in [7] we use a Bayesian fitting method [14] to fit the $3 \times 3$ matrix of hadron correlators to a multi-exponential form to extract the energies of states appearing in that correlator. This alows us to fit the entire correlator (i.e. for all time separations between source and sink), so making use of all the information contained in it. It also means that the fit results we obtain, for example for the ground state, include the effect of the higher excited states that are present in the correlator, and are not biassed by an attempt to fit only one or two states in a particular time window. The fitting function is

$$
G_{\text {meson }}\left(n_{s c}, n_{s k} ; t\right)=\sum_{k=1}^{n_{\text {exp }}} a\left(n_{s c}, k\right) a^{*}\left(n_{s k}, k\right) e^{-E_{k} t} .
$$

where $a\left(n_{s c / s k}, k\right)$ are the (real) amplitudes for state $k$ to appear in the smearings used at the source and sink of the correlator respectively.
The Bayesian fitting method [14] allows a large number of exponentials to be used in the fit by constraining the way in which these exponentials can appear based on physical information. The simplest physical information is that the energies of states are ordered, and we implement this in the fit by taking the energy fit parameters as the natural logarithms of the ground state energy and of the energy splittings between adjacent states. On top of this we apply priors to the splittings between adjacent states that constrain them to be of order $500 \mathrm{MeV}$ with a width of a factor of two, i.e. between $250 \mathrm{MeV}$ and $1000 \mathrm{MeV}$. Amplitudes are typically constrained around zero with a width of 1.0 (our composite meson smearing functions are normalised so that the spatial sum of their square is 1). We apply a cut on the range of eigenvalues present in the correlation matrix of $10^{-3}$ except for the high statistics calculation on the coarse 005/05 lattices where we use $10^{-4}$. This reduces the number of degrees of freedom in the fit to between 120 and 170, with 208 in the coarse $005 / 05$ fit. We obtain values for the $\Upsilon$ ground state energy and that of the first radial excitation, the $\Upsilon^{\prime}$ as a function of the number of exponentials in the fits. We demand a good $\chi^{2}$ and that the fit for 3 adjacent exponentials should agree both on the fitted values for the energies of interest and on the errors. The ground state energy stabilises very quickly, but the first excited state is not generally stable until we reach 8 exponentials. Fit results on the different MILC ensembles are then tabulated in Table III from 10 exponential fits.

Figure 1 shows results from our highest statistics calculation on the coarse 005/05 lattices. Here we are able to obtain a good signal for even higher excited states than the $2 S$. The plot shows the ratio of the $3 S-1 S$ splitting and the $4 S-1 S$ splitting to that of the $2 S-1 S$. The $3 S-1 S$ splitting is obtained to $3 \%$ and in agreement with experiment. The $4 S-1 S$ splitting is not very accurate even with the statistics available here. The result is slightly higher than experiment, but the $4 S$ state is not gold-plated, decaying to $B \bar{B}$. This is not taken account of accurately in the lattice calculation and so we expect our result to be higher than experiment. In our lower statistics calculations we do not generally have a significant signal for the $4 S$ and our $3 S-1 S$ splitting has an error of between $5 \%$ and $10 \%$.

As discussed earlier, the excitation energies for bound states of heavy quarks are almost independent of the heavy quark mass, meaning that accurate tuning of this mass is not required for these splittings. Use of the random wall does, however, allow us to determine the meson energy as a function of meson momentum much more accurately than in previous calculations, and so the meson 'kinetic' masses can be well determined. The meson mass in NRQCD must be determined from the meson dispersion relation because the zero of energy has an offset. The mass is then given by the difference in energy between mesons at zero momentum and momentum $p a$ on 


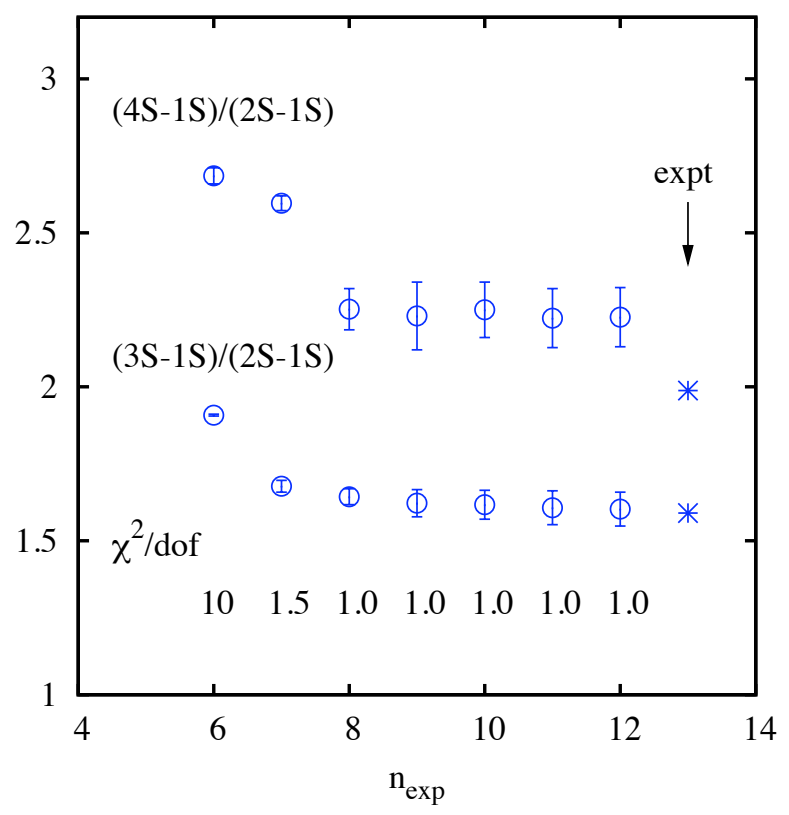

FIG. 1: Results for highly excited states from our fit to the $3 \times 3$ matrix of $\Upsilon$ correlators from the coarse 005/05 (set 4) ensemble as a function of the number of exponentials included in the fit. The $\chi^{2} /$ dof is also shown - the fit had 208 degrees of freedom. The results are stable from 9 to 12 exponentials.

the lattice by [7]:

$$
M a=\frac{p^{2} a^{2}-(\Delta E a)^{2}}{2 \Delta E a} .
$$

$\Delta E a$ is calculated by taking the difference in energy of the ground state from a simultaneous fit to $l l \Upsilon$ correlators made from a standard (zero momentum) random wall as described above, and a random wall patterned with an appropriate Fourier factor to give a momentum of $(1,0,0)$ to both quark and antiquark, so that the $\Upsilon$ has momentum $(2,0,0)$ (and its permutations). Values obtained for the kinetic mass on each of the MILC ensembles are given in Table III. They are tuned within $10 \%$ of the experimental result of $9.46 \mathrm{GeV}$ [15].

Table III gives results for the lattice spacings $a^{\Upsilon}$ obtained by dividing the simulation results for $a\left(E_{2}-E_{1}\right)$ by the experimental value of $0.5630 \mathrm{GeV}$ for the splitting. Statistical errors are at the level of 1\%. Systematic errors arise from two sources, discretisation errors and missing higher-order relativistic correctiosn to the NRQCD action. The former can be removed by continuum extrapolation as long as they are well-behaved. The leading discretisation corrections come from radiative corrections to existing terms in the action and can be calculated in perturbation theory. They have been shown to be small corrections in the region of $a M_{b}$ in which we work, and relatively independent of $a M_{b}$ [16]. Relativistic corrections survive the continuum limit and are the main source of systematic error for this method. They
TABLE III: Results for the ground state energy, $a E_{1}$, and radial excitation energy, $a\left(E_{2}-E_{1}\right)$ obtained from 10 exponential fits of the form in equation 6 to a $3 \times 3$ matrix of $\Upsilon$ correlators as described in the text. The 4 th column gives the $\Upsilon$ mass as determined from eq. 7. Fewer configurations were used for this than for the full calculation (and given in Table II in several cases. For set 3, 202 configurations were used and for set 8, 470 configurations. The 5 th column gives the result for the lattice spacing from setting the $2 S-1 S$ splitting equal to the experimental value of $0.5630 \mathrm{GeV}$ [15].

\begin{tabular}{lllll}
\hline \hline Set & $a E_{1}$ & $a\left(E_{2}-E_{1}\right)$ & $a M$ & $a^{\Upsilon} / \mathrm{fm}$ \\
\hline 1 & $0.28775(8)$ & $0.4244(33)$ & $7.226(12)$ & $0.1488(12)$ \\
2 & $0.28814(8)$ & $0.4309(32)$ & $7.231(12)$ & $0.1510(11)$ \\
\hline 3 & $0.29330(3)$ & $0.3439(8)$ & $5.983(10)$ & $0.1205(3)$ \\
4 & $0.29261(6)$ & $0.3462(38)$ & $5.985(11)$ & $0.1213(13)$ \\
\hline 6 & $0.26618(5)$ & $0.2381(37)$ & $4.281(12)$ & $0.0835(13)$ \\
\hline 8 & $0.24850(3)$ & $0.1679(14)$ & $3.050(18)$ & $0.0588(5)$ \\
\hline \hline
\end{tabular}

were estimated in [7] at $0.7 \%$ on the coarse and $0.6 \%$ on the fine lattices, so we include an overall systematic error of $0.7 \%$ in our error analysis here.

One ingredient missing from our calculation and present in the experimental world is electromagnetism. This is then another possible source of systematic error. From a potential model calculation we estimate the shift in the $2 S-1 S$ splitting to be less than $1 \mathrm{MeV}$ from the Coulomb interaction between $b$ and $\bar{b}$ (the electromagnetic self-interaction is included in the $b$ quark mass). At less than $0.2 \%$, this is negligible.

To extract a physical value for the static-quark potential parameter $r_{1}$, we must combine the lattice spacings $a_{i}^{\Upsilon}$ in Table III with the corresponding values of $\left(r_{1} / a\right)_{i}$ in Table I] and extrapolate to zero lattice spacing, correcting the sea-quark masses. We do this by fitting $\left(r_{1} / a\right)_{i} a_{i}^{\Upsilon}$ from the $i$ th ensemble to a formula for the effective $r_{1}$ corresponding to $m_{\Upsilon^{\prime}}-m_{\Upsilon}$ :

$$
\begin{aligned}
r_{1}^{\Upsilon}\left(a, \delta m_{l}^{\text {sea }},\right. & \left.\delta m_{s}^{\text {sea }}\right)=r_{1} \\
& \times\left(1+c_{\text {sea }}^{\Upsilon} \frac{2 \delta m_{l}^{\text {sea }}+\delta m_{s}^{\text {sea }}}{m_{s}}\right) \\
& \times\left(1+\sum_{j=1}^{4} c_{j}^{\Upsilon}\left(a / r_{1}\right)^{2 j}\right),
\end{aligned}
$$

where $r_{1}$ (the extrapolated value), $c_{\text {sea }}^{\Upsilon}$ and $c_{j}^{\Upsilon}$ are the parameters tuned by the fit. Here the $\delta m^{\text {sea }}$ are differences between the sea-quark masses used in the simulation and the correct masses for $l=u / d$ and $s$ quarks (see Appendix C).

We have included twice as many terms as we need in the expansion in $a / r_{1}$; taking half as many terms gives essentially identical results. We are able to retain higherorder terms because we include Bayesian priors in our fit for each expansion coefficient used - that is, we include an initial estimate for each parameter. Each prior functions as an additional piece of input data in the fit, thereby guaranteeing that we always have more fit data 
TABLE IV: Major sources of uncertainty in physical $r_{1}$ values obtained from simulation results for $m_{\Upsilon^{\prime}}-m_{\Upsilon}, m_{D_{s}}-m_{\eta_{c}} / 2$, $f_{\eta_{s}}$, and from an analysis that fits all three types of data simultaneously.

\begin{tabular}{rllll}
\hline \hline & $\Upsilon$ & $D_{s}$ & $f_{\eta_{s}}$ & combined \\
\hline$a^{2}$ extrapolation & $0.4 \%$ & $0.8 \%$ & $0.1 \%$ & $0.2 \%$ \\
$m_{s}$ extrapolations & - & 0.0 & 0.0 & 0.0 \\
$r_{1} / a$ uncertainty & 0.2 & 0.2 & 0.1 & 0.1 \\
initial uncertainty in $r_{1}$ & 0.6 & 0.8 & - & - \\
$\pi-K-\eta_{s}$ analysis & - & - & 0.8 & 0.6 \\
statistical errors & 1.1 & 0.4 & 0.2 & 0.3 \\
sea-quark mass tuning & 0.1 & 0.2 & 0.0 & 0.1 \\
overall systematic error & 0.6 & 1.1 & - & 0.2 \\
\hline Total & $1.4 \%$ & $1.6 \%$ & $0.9 \%$ & $0.7 \%$ \\
\hline \hline
\end{tabular}

than parameters, no matter how many parameters we choose to keep. In Bayesian fits like ours it is important to keep more parameters rather than fewer, not because they improve the fit but rather because they help us avoid underestimating our extrapolation errors. Here we used priors $c_{j}^{\Upsilon}=0(1), c_{\mathrm{sea}}^{\Upsilon}=0.0(1)$, both of which are broader (i.e., more conservative) than suggested by the empirical Bayes criterion [14. Setting $c_{\text {sea }}^{\Upsilon}$ to zero has negligible impact, so this parameter is not really necessary. We also take a very broad prior for $r_{1}$ that encompasses all current estimates: $r_{1}=0.315(10) \mathrm{fm}$. It has little impact on our final errors.

Fitting our data, we obtain a final value for the physical $r_{1}$ from the upsilon simulations of:

$$
r_{1}=0.3091(44) \mathrm{fm} \quad\left(\text { from } m_{\Upsilon^{\prime}}-m_{\Upsilon}\right) .
$$

The fit is excellent, with a $\chi^{2}$ per degree of freedom of 0.2 . We show plots in Section III. The main sources of error in this result are listed in the $\Upsilon$-column of Table IV] most of the error is due to statistical errors from the Monte Carlo simulation. Improvement will require much higher statistics on the fine and superfine lattices.

$$
\text { B. } m_{D_{s}}-m_{\eta_{c}} / 2
$$

A useful mass difference in the charm sector is that between the $D_{s}$ meson and one half of the mass of a low-lying $c \bar{c}$ state. We choose the $\eta_{c}$ rather than the $J / \psi$ because it is the easiest for us to calculate. This splitting has the experimental value $0.4784(7) \mathrm{GeV} 15$ which changes to $0.672(2) \mathrm{GeV}$ when the charm quark is replaced by a bottom quark. So the sensitivity to the heavy-quark mass, while stronger than for the heavyonium splittings, is still rather mild. Both the $D_{s}$ and the $\eta_{c}$ are ground state mesons and do not have the poor signal/noise issues that the $\Upsilon(2 S)$ state had in the previous subsection. Two quark masses are involved in the $D_{s}-\eta_{c} / 2$ splitting, however, and this makes the tuning rather complicated. As a result we have only done this on two ensembles - coarse set 4 (see Table I) using 595 configurations with 2 time sources for propagators on each

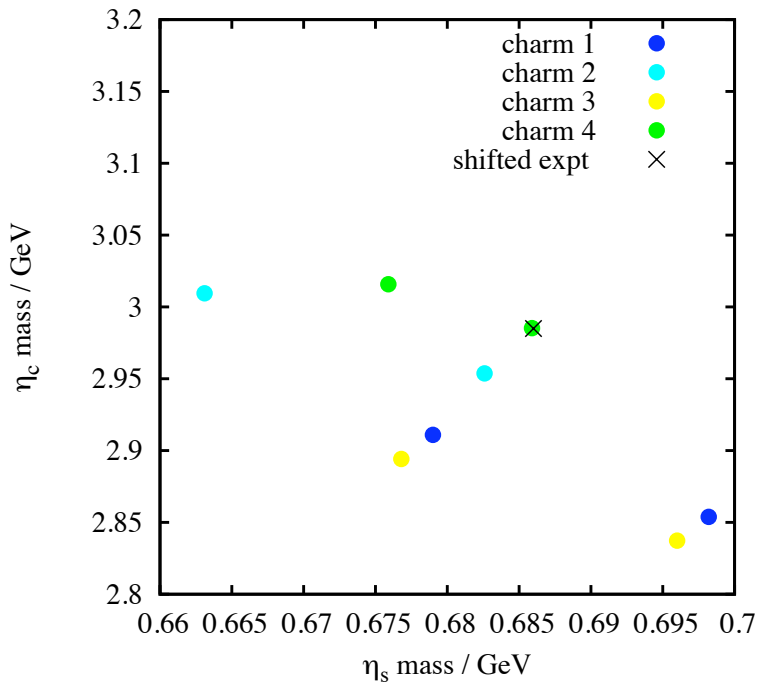

FIG. 2: Results for $m_{\eta_{c}}$ vs $m_{\eta_{s}}$ for different charm and strange quark masses on the coarse 01/05 ensemble (set 4). Points corresponding to different charm quark masses are given in different colors. Several different strange quark masses are given for each charm quark mass. The lattice spacing is determined from $m_{D_{s}}-m_{\eta_{c}} / 2$. Note that the experimental point is shifted to allow for electromagnetic effects missing from our calculation, as described in the text.

configuration and fine set 6 using 566 configurations with 4 time sources each.

Following the development of the HISQ action [17] it is now possible to handle charm quarks accurately with a relativistic action in lattice QCD. The success of this action is demonstrated by an accurate $(7 \mathrm{MeV})$ determination, that agrees with experiment, of the masses of $D$ and $D_{s}$ meson when the charm quark mass is fixed from the $\eta_{c}$ [1]. This has not been possible with any other discretisation of QCD for charm quarks. Here we are essentially inverting this calculation to use $m_{D_{s}}-m_{\eta_{c}} / 2$ to determine a value for the lattice spacing, simultaneously requiring that the mass of the $\eta_{c}$ and the $\eta_{s}$ (the fictitious pseudoscalar particle made of an $s$ quark-antiquark pair, see subsection C) be correct. We use the HISQ action as described in 17. except that we simplify the tuning of the coefficient of the Naik term (that corrects for $a^{2}$ errors) so that it is correct as a function of $m a$ at tree level. In [17] it was shown that a nonperturbative tuning of this coefficient gave results very similar to the tree-level result and so it is much simpler to take the tree-level result at each value of the quark mass. The difference between tree-level and nonperturbative tuning of the coefficient is a small discretisation error at relatively high order, and it will be taken care of in our continuum extrapolation.

We proceed by calculating $\eta_{c}, \eta_{s}$ and $D_{s}$ correlators for several different combinations of bare quark masses for charm and strange quarks. We use random wall sources as for the $b$ quarks in the previous subsection, which improves the statistical error on the ground state masses 
that we need here significantly. No smearing function is necessary. Each of the correlators is fit to an appropriate multi-exponential form including oscillating states for the $D_{s}[1]$.

$$
G_{\text {meson }}(t)=\sum_{k=1}^{n_{\text {exp }}} a_{k}\left(e^{-M_{k} t}+e^{-M_{k}(T-t)}\right),
$$

for $\eta_{c}$ and $\eta_{s}$ and

$$
\begin{aligned}
G_{\text {meson }}(t) & =\sum_{k=1}^{n_{\text {exp }}} a_{k}\left(e^{-M_{k} t}+e^{-M_{k}(T-t)}\right) \\
& +\sum_{k o=1}^{n_{\text {exp }}} a_{k o}(-1)^{t} a_{k o}\left(e^{-M_{k o} t}+e^{-M_{k o}(T-t)}\right)
\end{aligned}
$$

for $D_{s}$. As before, standard Bayesian fitting techniques are used [14 and results are taken from fits with $n_{\text {exp }}=4$, where we find the results, and their errors, are stable to changes in $n_{\text {exp }}$.

For each combination it is then possible to plot the value of $m_{\eta_{c}}$ against that of $m_{\eta_{s}}$ using the lattice spacing from $m_{D_{s}}-m_{\eta_{c}} / 2$ and interpolate to experiment. A plot showing our results on the coarse 01/05 (set 4) ensemble is given in Figure 2. Interpolation to the matching point is relatively simple because the $D_{s}$ mass is linear in $m_{s} a$ and $m_{c} a$, the $\eta_{c}$ mass is linear in $m_{c} a$ and the square of the $\eta_{s}$ mass is linear in $m_{s} a$ for small changes in the quark masses. As can be seen from the Figure it is possible to pinpoint the matching point precisely and then confirm it with an additional calculation. Statistical errors below $0.5 \%$ are achievable.

The experimental values to be used here must take into account that the lattice calculation is missing the electromagnetism of the real world. This gives a significant shift to $m_{D_{s}}-m_{\eta_{c}} / 2$ because the $D_{s}$ is electrically charged and the $\eta_{c}$ is neutral, so their masses shift in opposite directions and the shifts add together. We estimate the electromagnetic shift to the $D_{s}$ mass to be 1.4 MeV, and the shift to the $\eta_{c}$ mass to be $-2.6 \mathrm{MeV}$. In addition the $\eta_{c}$ in the real world can annihilate to gluons but not in our calculation and we estimate the shift from this effect to be $-2.4 \mathrm{MeV}$ [17]. This shifts $m_{D_{s}}-m_{\eta_{c}} / 2$ from the real world to $0.4745 \mathrm{MeV}$ for comparison to our calculation. We take the systematic error on this value to be one half of the shift we apply: that is, $2 \mathrm{MeV}$. This $0.5 \%$ error gives a $1.5 \%$ systematic error on the values of the lattice spacing that we obtain, unfortunately dominating our statistical errors. The experimental mass for the $\eta_{c}$ becomes $2.985 \mathrm{GeV}$ after applying the shifts above and the $\eta_{s}$ mass is taken as $0.686 \mathrm{GeV}$ (see Appendix A and Eq. (A6).

Table V gives results on the coarse (set 4) and fine (set 6 ) lattices from this method. The $\eta_{s}$ correlators are a subset of those used in subsection C. The results for $m_{\eta_{s}}$ are very slightly different in the two cases because of the different fitting strategy employed.

Given the lattice spacings in Table V, we can again combine them with the corresponding values for $r_{1} / a$
TABLE V: Results for $\Delta=m_{D_{s}}-m_{\eta_{c}} / 2$ in lattice units from different charm and strange HISQ quark masses on ensembles 4 and 6 . The corresponding lattice spacing, at the tuned point where $m_{\eta_{c}}$ and $m_{\eta_{s}}$ agree with their physical values, are given

\begin{tabular}{|c|c|c|c|c|c|}
\hline \multicolumn{6}{|l|}{ set 4} \\
\hline$m_{c} a$ & $m_{\eta_{c}} a$ & $m_{s} a$ & $m_{\eta_{s}} a$ & $a \Delta$ & \multirow[t]{4}{*}{$a / \mathrm{fm}$} \\
\hline 0.72 & $1.98114(15)$ & 0.06 & $0.45787(23)$ & $0.3180(5)$ & \\
\hline 0.753 & $2.04293(10)$ & 0.06 & $0.45787(23)$ & $0.3214(5)$ & \\
\hline 0.753 & $2.04293(10)$ & 0.063 & $0.46937(24)$ & $0.3247(5)$ & \\
\hline \multicolumn{4}{|c|}{ tuned } & $0.3247(5)$ & $0.1350(2)$ \\
\hline \multicolumn{6}{|l|}{ set 6} \\
\hline$m_{c} a$ & $m_{\eta_{c}} a$ & $m_{s} a$ & $m_{\eta_{s}} a$ & $a \Delta$ & \multirow[t]{5}{*}{$a / \mathrm{fm}$} \\
\hline 0.44 & $1.33816(7)$ & 0.0358 & $0.30332(12)$ & $0.21244(23)$ & \\
\hline 0.44 & $1.33816(7)$ & 0.0382 & $0.31362(14)$ & $0.21535(22)$ & \\
\hline 0.45 & $1.35934(7)$ & 0.0358 & $0.30332(12)$ & $0.21350(24)$ & \\
\hline \multirow[t]{2}{*}{0.45} & $1.35934(7)$ & 0.0382 & $0.31362(14)$ & $0.21640(23)$ & \\
\hline & tuned & & & $0.2174(5)$ & $0.0904(2)$ \\
\hline
\end{tabular}
in the bottom row of each section of the table. Errors shown are from statistics and extrapolation only.

from Table I to obtain effective values $r_{1}^{D_{s}}$ that we can extrapolate to zero lattice spacing. We do this using the same parameterization and priors for $r_{1}^{D_{s}}$ as we did for $r_{1}^{\Upsilon}$ in the previous section, except that here we allow for less dependence on the sea-quark masses since that is what our previous simulations have shown [1]. We take $c_{\text {sea }}=0.00(1)$ as a prior. With only two data points, the fit is almost trivial, giving

$$
r_{1}=0.3157(53) \mathrm{fm} \quad\left(\text { from } m_{D_{s}}-m_{\eta_{c}} / 2\right),
$$

which agrees well with our estimate from the $\Upsilon$ but is less accurate. The main sources of error in this result are listed in the $D_{s}$-column of Table IV; the largest source of error is the overall systematic error [18. The systematic error could be improved slightly by using the $J / \psi$ instead of the $\eta_{c}$ to avoid the sizeable mass shift from annihilation to gluons and its uncertainty. In addition a more accurate understanding of electromagnetic mass shifts, with quantitative tests on the lattice would help (see, for example, [19]).

$$
\text { C. } f_{\eta_{s}}
$$

The $\eta_{s}$ is a fictitious pseudoscalar meson. It is like the pion and kaon, but with valence $s \bar{s}$ quarks. In the real world the valence $s \bar{s}$ state mixes with $u \bar{u}$ and $d \bar{d}$, through valence quark-antiquark annihilation, to form the $\eta$ and $\eta^{\prime}$ mesons. By omitting valence quark-antiquark annihilation from our simulation, we obtain the $\eta_{s}$ instead. This meson is easily studied, in lattice QCD, using simulations and, in the continuum, using partially-quenched chiral perturbation theory 20. In Appendix A we show how to determine its mass and decay constant from simulation and experimental data for pions and kaons, using chiral perturbation theory. We are able to determine both parameters to within about $0.5 \%$. 
TABLE VI: Simulation results for the $\eta_{s}$ mass $m_{\eta_{s}}$ and decay constant $f_{\eta_{s}}$ for several lattice parameter sets (see Table I) and $s$-quark masses $a m_{s}$. We also list the number of gauge field configurations and time sources per configuration used.

\begin{tabular}{clccc}
\hline \hline Set & $a m_{s}$ & $a f_{\eta_{s}}$ & $a m_{\eta_{s}}$ & $n_{c f g} \times n_{t}$ \\
\hline 1 & 0.066 & $0.1429(4)$ & $0.5250(6)$ & $631 \times 2$ \\
& 0.08 & $0.1485(4)$ & $0.5782(6)$ & $631 \times 2$ \\
2 & 0.066 & $0.1436(4)$ & $0.5248(6)$ & $631 \times 2$ \\
\hline 3 & 0.0537 & $0.1144(2)$ & $0.4310(4)$ & $518 \times 2$ \\
4 & 0.0546 & $0.1160(3)$ & $0.4367(5)$ & $595 \times 2$ \\
& 0.05465 & $0.1160(3)$ & $0.4369(5)$ & $595 \times 2$ \\
& 0.06 & $0.1182(4)$ & $0.4580(5)$ & $595 \times 2$ \\
5 & 0.0525 & $0.1149(4)$ & $0.4259(6)$ & $460 \times 2$ \\
& 0.0556 & $0.1161(4)$ & $0.4384(6)$ & $460 \times 2$ \\
\hline 6 & 0.0358 & $0.0806(2)$ & $0.3035(3)$ & $566 \times 4$ \\
& 0.0366 & $0.0810(2)$ & $0.3069(3)$ & $566 \times 4$ \\
& 0.0382 & $0.0817(2)$ & $0.3137(3)$ & $566 \times 4$ \\
7 & 0.03635 & $0.0811(2)$ & $0.3050(4)$ & $265 \times 4$ \\
\hline 8 & 0.024 & $0.0556(1)$ & $0.2120(2)$ & $218 \times 4$ \\
\hline 9 & 0.0165 & $0.0408(1)$ & $0.1548(1)$ & $200 \times 2$ \\
& 0.018 & $0.0417(2)$ & $0.1621(2)$ & $101 \times 1$ \\
\hline \hline
\end{tabular}

Given an accurate physical value, the $\eta_{s}$ mass is the easiest quantity to use for tuning the $s$-quark mass in lattice simulations. It is significantly simpler to use than the $K$ mass since $m_{\eta_{s}}$, unlike $m_{K}$, is only weakly dependent upon the $u / d$ mass and therefore requires only minimal chiral extrapolation. This is because $u / d$ quarks enter only in the sea for this meson. Another advantage of the $\eta_{s}$ is that it is much less expensive to simulate than the $K$.

Given a tuned $s$-quark mass, $f_{\eta_{s}}$ is much more useful for tuning the lattice spacing than either $f_{K}$ or $f_{\pi}$. Again, this is because it is almost independent of the $u / d$ mass (and because it is much less expensive to compute). We have computed both the decay constant and the mass for the $\eta_{s}$ for a variety of $s$-quark masses for all of our lattice parameter sets. The results are given in Table VI.

As discussed earlier, we used the HISQ formalism for the valence $s$ quarks in our analysis, together with the MILC gluon configurations described in Table [] We analyzed the $\eta_{s}$ created by the partially conserved axialvector current in the HISQ formalism, so that the decay constant is automatically correctly normalized, with no need for further renormalization constants. We used random-wall sources when computing quark propagators, as described earlier for $b$-quark propagators, and used sources on several time slices for each configuration, to increase statistics, see Table VI.

We extracted masses and decay constants from the meson correlators by fitting the middle $40 \%$ of the $t$ range to a single exponential. This is less sophisticated than our approach to fitting correlators in previous subsections, but it simplifies the analysis of statistical correlations between different results coming from the same ensemble (with different $s$-quark masses). We get identical results if we use instead results from multi-exponential fits, ig- noring correlations. The $\chi^{2}$ per degree of freedom of our fits was larger than one for some ensembles, possibly because of lower statistics. To be conservative, we doubled the statistical errors everywhere (giving the results in Table VI, resulting in excellent $\chi^{2}$ s.

In analyzing our simulation results for $\left(a f_{\eta_{s}}\right)_{i}$ and $\left(a m_{\eta_{s}}\right)_{i}$, we need to account for three systematic effects. First none of the simulations has precisely the correct $s$ quark mass $m_{s}$. We did simulations at multiple values of $m_{s}$ so that we could interpolate. The lattice spacing cancels out in the ratio $\left(a f_{\eta_{s}}\right)_{i} /\left(a m_{\eta_{s}}\right)_{i}$; we in effect vary $m_{s}$ until this ratio has the correct continuum value, obtained from our chiral analysis (see Appendix A.

The second important systematic effect is that our simulations have finite-lattice-spacing errors. We model dependence on the lattice spacing using a power series in $\left(a / r_{1}\right)^{2}$. A final, but much less important systematic is that the sea-quark masses are not quite right in our simulations. We did simulations using several different sea-quark masses so that we could correct for this dependence, which, as discussed above, we expect (and find) to be very small. Other systematic errors are negligible. In particular, finite-volume corrections for the $\eta_{s}$ are no larger than $0.1 \%$ in our simulations.

We account for these systematic effects by fitting our results $\left(a f_{\eta_{s}}\right)_{i}$ from the simulation using ensemble set $i$, with each $s$ mass, to:

$$
\left(a / r_{1}\right)_{i} r_{1}^{\eta_{s}} f_{\eta_{s}}^{\text {lat }}\left(a_{i}, x_{\eta_{s}}\right)
$$

where again values for $\left(r_{1} / a\right)_{i}$ come from Table I] This formula defines $r_{1}^{\eta_{s}}$, which is the effective value of $r_{1}$ implied (for each ensemble set) by our data for the $\eta_{s}$ decay constant and mass. We parameterize $r_{1}^{\eta_{s}}$ the same way we parameterized $r_{1}^{\Upsilon}$ and $r_{1}^{D_{s}}$ :

$$
\begin{aligned}
r_{1}^{\eta_{s}}\left(a, \delta m_{l}^{\text {sea }},\right. & \left.\delta m_{s}^{\text {sea }}\right)=r_{1} \\
& \times\left(1+c_{\text {sea }}^{\eta_{s}} \frac{2 \delta m_{l}^{\text {sea }}+\delta m_{s}^{\text {sea }}}{m_{s}}\right) \\
& \times\left(1+\sum_{j=1}^{4} c_{j}^{\eta_{s}}\left(a / r_{1}\right)^{2 j}\right)
\end{aligned}
$$

where again $r_{1}$ is the physical value. Function $f_{\eta_{s}}^{\text {lat }}\left(a, x_{\eta_{s}}\right)$ models the $s$-quark mass dependence of the decay constant where

$$
x_{\eta_{s}} \equiv\left(\frac{\left(a m_{\eta_{s}}\right)_{i}}{\left(a f_{\eta_{s}}\right)_{i}} \frac{f_{\eta_{s}}}{m_{\eta_{s}}}\right)^{2}-1
$$

is a measure of difference between the correct $s$ mass and the $s$ mass used in the simulation to produce $\left(a f_{\eta_{s}}\right)_{i}$ and $\left(a m_{\eta_{s}}\right)_{i}$. We parameterize $f_{\eta_{s}}^{\text {lat }}$ as follows:

$$
f_{\eta_{s}}^{\text {lat }}\left(a, x_{\eta_{s}}\right)=f_{\eta_{s}}+\sum_{k=1}^{4} d_{k} x_{\eta_{s}}^{k} .
$$

We allow the first two terms in the expansion to depend upon the lattice spacing by taking

$$
d_{k} \equiv d_{k 0}+d_{k 1}\left(a / r_{1}\right)^{2}
$$


for $j=1,2$; lattice-spacing dependence in the higherorder terms would have negligible effect (as do the higherorder terms themselves, as it turns out).

Again we have included twice as many terms as we need in the expansions in $a / r_{1}$ and $x_{\eta_{s}}$; taking half as many terms in both cases gives essentially identical results. Here we used priors $c_{j}^{\eta_{s}}=0(1)$ and $c_{\mathrm{sea}}^{\eta_{s}}=0.0(1)$, as before, and $d_{k l}=0.0(5)$. Again all priors are somewhat broader (that is, more conservative) than suggested by the empirical Bayes criterion 14 .

The other parameter varied in the fit is the continuum/physical $r_{1}$ in Eq. (14). We tried two different priors for this parameter. First we took the very broad prior, $0.315(10) \mathrm{fm}$, we used for the other quantities. We also fit using the $r_{1}$ result from our chiral analysis of $f_{\pi}$ and $f_{K}$ in Appendix A (Eq. (A6)). These two choices give results that differ by only a tenth of a standard deviation, which is negligible. We use the latter choice for our results below. We also take the values for $f_{\eta_{s}}$ and $\left(f_{\eta_{s}} / m_{\eta_{s}}\right)$ used in Eqs. (16) and (15) from our chiral analysis as described in Appendix A

Our final result for the continuum value for $r_{1}$ in this analysis is:

$$
r_{1}=0.3148(28)(5) \text { fm } \quad\left(\text { from } f_{\eta_{s}}\right),
$$

where, as discussed in Appendix A the second error corresponds to uncertainty about finite-volume corrections in the chiral analysis. The fit is excellent, with a $\chi^{2}$ per degree of freedom of 0.4. The main sources of error in this result are listed in the $f_{\eta_{s}}$-column of Table IV] the largest source of error is uncertainty in the physical values of $f_{\eta_{s}}$ and $m_{\eta_{s}}$ from the $\pi-K-\eta_{s}$ chiral analysis.

\section{TWO RECIPES}

Two accurate recipes for setting the lattice spacing follow from the analysis in the previous section. The first requires that the static-quark potential be computed in the simulation, and a value for $r_{1} / a$ extracted from the results. This has been done accurately by the MILC collaboration for their ensembles and we use their numbers. $r_{1} / a$ can then be converted to a value for the lattice spacing by dividing into the physical value of $r_{1}$. In the previous section, we did separate determinations of $r_{1}$ using simulation results for the upsilon and $D_{s}$ mass splittings, and for the $\eta_{s}$ decay constant. For each we extracted effective values of $r_{1}$ for each lattice ensemble and parameter set; and we extrapolated to the continuum to obtain physical values for $r_{1}$. We have also done a joint analysis of all three sets of simulation results which is identical to what we did for each separately, but requiring that each fit use the same physical $r_{1}$ - that is, we require all three to agree on the final value for $r_{1}$. This analysis also implicitly includes the $r_{1}$ result from our chiral analysis of $f_{\pi}$ and $f_{K}$ since we use that value as the input prior for the combined analysis. When we do this we obtain the following final result, where again the second error

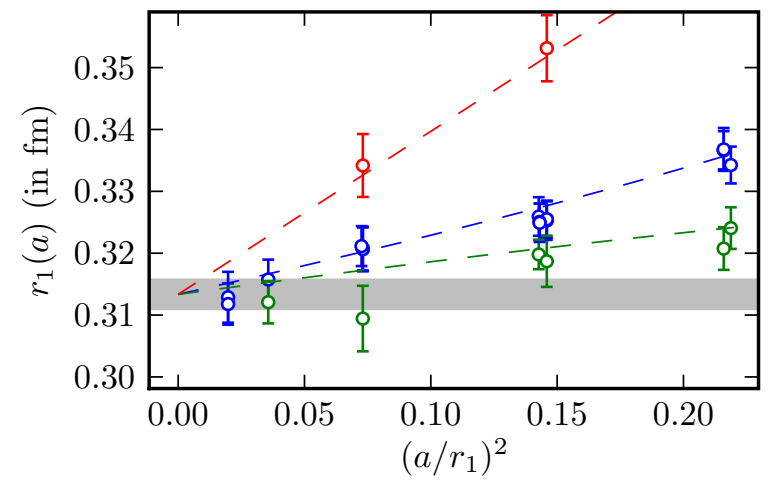

FIG. 3: Simulation results for the effective $r_{1}$ obtained from $m_{D_{s}}-m_{\eta_{c}} / 2$ (top), $f_{\eta_{s}}$ (middle), and $m_{\Upsilon^{\prime}}-m_{\Upsilon}$ (bottom) are plotted versus $\left(a / r_{1}\right)^{2}$ for various values of the sea-quark mass. The lines show the tuned fit functions from our simultaneous fit to all three sets of simulation results. We used the fit functions to correct the simulation data points for the sea-quark masses; data points and lines are for $\delta m_{q}^{\text {sea }}=0$. The gray band is the continuum value obtained from the fit: $r_{1}=0.3133(23) \mathrm{fm}$.

is due to uncertainties in finite-volume corrections to the chiral analysis (see Appendix A):

$$
r_{1}=0.3133(23)(3) \mathrm{fm} \quad \text { (combined) }
$$

The fit is excellent with a $\chi^{2}$ per degree of freedom of 0.4 . Figure 3 shows $r_{1}$ values from all three simulations plotted against the square of the lattice spacing. The sources of error in this combined analysis are summarized in the last column of Table IV The $a^{2}$ dependence in that figure is all relative to $a^{2}$ dependence in the $r_{1} / a$ values obtained from the static-quark potential. Thus the upsilon analysis has $a^{2}$ errors most similar to those in the staticquark potential's $r_{1} / a$, while the $D_{s}$ analysis has errors least like those coming from the static-quark potential. There is no way to tell which of these quantities has the smallest absolute finite- $a$ errors from just this simulation data; all that we can say is that they are consistent with each other in the continuum limit.

The second recipe for determining the lattice spacing for a particular configuration set requires only the evaluation of the mass and decay constant for the $\eta_{s}$ (see Section II C on those configurations; there is no need for the static-quark potential in this recipe. Lattice results for $a f_{\eta_{s}}$ are fit to the formula

$$
a f_{\eta_{s}}^{\text {lat }}=a f_{\eta_{s}}\left(1+c_{1} x_{\eta_{s}}+c_{2} x_{\eta_{s}}^{2}\right),
$$

where, as before,

$$
x_{\eta_{s}}=\left(\frac{f_{\eta_{s}}}{m_{\eta_{s}}} \frac{a m_{\eta_{s}}^{\mathrm{lat}}}{a f_{\eta_{s}}^{\text {lat }}}\right)^{2}-1,
$$

and $a, c_{1}$, and $c_{2}$ are fit parameters. Physical values for the mass and decay constant, $m_{\eta_{s}}$ and $f_{\eta_{s}}$, are again 
taken from Eqs. A6 in Appendix A Our simulations indicate that $c_{1}=0.33(5)$ and $c_{2}=0.0(5)$ are good priors for these parameters; further terms in the $x_{\eta_{s}}$ expansion are unnecessary provided $x_{\eta_{s}}$ is small (it is less than 0.06 for our data). The lattice spacing for the particular configuration set under study is then an output from the fit.

In a typical simulation one guesses a value for the bare $s$-quark mass, $a m_{s}$ in lattice units, to use in the quark action. Provided this is close enough to the correct value, a fit of the $\eta_{s}$ results from this single mass is enough to generate an accurate lattice spacing. Doing simulations with two or more $s$-quark masses improves the result.

The correct value for $a m_{s}$ can also be estimated using a formula similar to Eq. 20. A simpler procedure that gives almost identical results (to within $1 / 4 \%$ ) for the correctly tuned $s$ mass uses

$$
a m_{s}^{\text {tuned }} \approx a m_{s}\left(\frac{m_{\eta_{s}}}{a m_{\eta_{s}}^{\text {lat } / a}}\right)^{2}
$$

where the lattice spacing is obtained from one of the two recipes above (or any other). Again in typical simulations, one guesses a value for $a m_{s}$ and then uses $\eta_{s}$ results for this mass, together with this formula, to refine the initial guess.

We compare lattice spacings determined using each of our two recipes in Table VII. As expected, the lattice spacings are very different on the coarser lattices. This is because $a^{2}$ errors differ between the $r_{1}$ and $\eta_{s}$ measurements. Also as expected (and required), the two recipes converge for smaller lattice spacings, as $a^{2}$ errors in both types of measurement become negligible. The errors in each case are comparable. We also include values for the correctly tuned $s$-quark mass (in the HISQ formalism) for each configuration set, and for each recipe for the lattice spacing.

In neither of our recipes do we attempt to correct for sea-quark masses that are not correctly tuned. This is standard practice in lattice determinations of the lattice spacing. It pushes any sea-quark mass dependence from $r_{1}$ or $f_{\eta_{s}}$ (or whatever is used to determine the lattice spacing) into the other measurements of interest. This is a small effect for $r_{1}$ and $f_{\eta_{s}}$, and it is typically extrapolated away together with the sea-quark effects intrinsic to the other measurements.

\section{IV. $r_{0}$}

$r_{0} / a$ is not determined directly by the MILC Collaboration. Instead they determine the coefficient of the $1 / r$ term in the static potential in the region $0.2-0.7 \mathrm{fm}$. If this coefficient is $B$ then:

$$
\frac{r_{0}}{r_{1}}=\sqrt{\frac{B+C_{r_{0}}}{B+C_{r_{1}}}}
$$

TABLE VII: Lattice spacings (in fm) and $s$-quark masses (in lattice units) determined using our $r_{1}$ and $f_{\eta_{s}}$ recipes. Results are given for each configuration set from Table I] We also list the number of $a m_{s}$ values used in the $\eta_{s}$ recipe. Note that the estimates converge as the lattice spacings vanish.

\begin{tabular}{cccccc}
\hline \hline Set & \#am & $\left.a\right|_{r_{1}}$ & $\left.a m_{s}^{\text {tuned }}\right|_{r_{1}}$ & $\left.a\right|_{\eta_{s}}$ & $\left.a m_{s}^{\text {tuned }}\right|_{\eta_{s}}$ \\
\hline 1 & 2 & $0.1456(11)$ & $0.0613(12)$ & $0.1583(13)$ & $0.0724(15)$ \\
2 & 1 & $0.1465(11)$ & $0.0622(12)$ & $0.1595(14)$ & $0.0736(16)$ \\
\hline 3 & 1 & $0.1184(9)$ & $0.0489(9)$ & $0.1247(10)$ & $0.0542(11)$ \\
4 & 3 & $0.1197(9)$ & $0.0495(9)$ & $0.1264(11)$ & $0.0553(11)$ \\
5 & 2 & $0.1185(9)$ & $0.0491(9)$ & $0.1263(11)$ & $0.0558(12)$ \\
\hline 6 & 3 & $0.0847(6)$ & $0.0337(6)$ & $0.0878(7)$ & $0.0362(7)$ \\
7 & 1 & $0.0844(6)$ & $0.0336(6)$ & $0.0884(7)$ & $0.0369(7)$ \\
\hline 8 & 1 & $0.0592(4)$ & $0.0226(4)$ & $0.0601(5)$ & $0.0233(5)$ \\
\hline 9 & 2 & $0.0440(3)$ & $0.0161(3)$ & $0.0443(4)$ & $0.0163(3)$ \\
\hline \hline
\end{tabular}

where $C_{r_{0}}=1.65$ and $C_{r_{1}}=1.0$ [3]. This assumes that the same constant $1 / r$ coefficient would be obtained around $r \approx r_{0}$ and $r \approx r_{1}$ and there will be a small systematic error, yet to be determined [5] for this assumption. $B$ shows dependence on the lattice spacing and the sea quark masses as demonstrated in Figure 13 of 5]. Extrapolating to the continuum and chiral limits gives $B=-0.464(7)$, implying from equation 23, with the caveats above, that $r_{0} / r_{1}=1.488(5)$. Our value for $r_{1}$ then gives $r_{0}=0.4661(38) \mathrm{fm}$. This is in agreement with, but more accurate than, the previous MILC determination of $0.462(12)$ fm which used ensembles at fewer values of the lattice spacing, but which includes a systematic error of 0.004 from the variation of results with fit range in $r$. Our result also agrees with the direct determination from Aoki et al [21] of $r_{0}=0.48(1)(1) \mathrm{fm}$, which also includes the effect of $u, d$ and $s$ sea quarks and comes from an analysis with multiple values of the lattice spacing.

\section{CONCLUSIONS}

The accurate determination of the lattice spacing is of critical importance to obtaining accurate results from lattice QCD. Here we give two ways to do this with sub$1 \%$ errors for the first time.

The first method makes use of the $\approx 0.3 \%$ accurate values for $r_{1} / a$ calculated by the MILC collaboration on their ensembles (which could also be reproduced on other ensembles with similar statistics) coupled with the $0.8 \%$ accurate value for $r_{1}$ given here : $r_{1}=0.3133(23) \mathrm{fm}$. Our result is $1.5 \sigma$ from our previous analysis [7] using only the $\Upsilon 2 S-1 S$ splitting on fewer ensembles and combined with less accurate $r_{1} / a$ values. It is also $1 \sigma$ lower than that of the MILC collaboration using essentially the same results [3. It is in agreement with, but slightly more accurate than a newer result from MILC [5] of $r_{1}=0.3108\left(\begin{array}{l}+30 \\ -80\end{array}\right)$ fm using $f_{\pi}$ data across a similar 
range of lattice spacing values to our $f_{\eta_{s}}$ analysis but with ASQTAD valence quarks rather than HISQ quarks [5].

The second method is possibly simpler (in the absence of $r_{1} / a$ values) since it relies only on a standard meson spectrum calculation that would automatically be included in many lattice analyses. The mass and the decay constant of the $\eta_{s}$ can be determined to better than $0.25 \%$ given similar statistics to those we have used here and provided a quark formalism is used in which the PCAC relation holds so that the decay constant has no renormalisation. Then the physical values for $f_{\eta_{s}}$ and $m_{\eta_{s}}$ that have been determined here can be used to find both the tuned value of the strange mass, by interpolation in $f_{\eta_{s}} / m_{\eta_{s}}$ to $0.2647(18)$ and the lattice spacing, from taking $f_{\eta_{s}}=0.1815(10) \mathrm{GeV}$ at the tuned point.

The two methods are compared for the MILC ensembles in Table VII.

To improve these methods so that errors below $0.5 \%$ are possible will require improvements in the chiral analysis determining the $\eta_{s}$ parameters. These can be gauged from the error budgets in Tables IV]and IX Key improvements that are certainly possible are statistical errors in the lattice results and accurate lattice data closer to the chiral and continuum limits. Improvements to other methods of determining the lattice spacing, such as that using the $\Upsilon$ spectrum and $m_{D_{s}}-m_{\eta_{c}} / 2$ discussed here are important for cross-checks of systematic effects.

Acknowledgements We are grateful to the MILC collaboration for the use of their configurations and to Doug Toussaint, Steve Gottlieb and Claude Bernard for information on $r_{1} / a$ values. We are grateful to other members of the HPQCD collaboration for useful discussions. Computing was done at USQCD's Fermilab cluster, the Ohio Supercomputer Centre and the Argonne Leadership Computing Facility at Argonne National Laboratory, which is supported by the Office of Science of the U.S. Department of Energy under constract DOE-AC0206CH11357. We acknowledge the use of Chroma [23] for part of our analysis. This work was supported by the Leverhulme Trust, MICINN, NSF, the Royal Society, the Scottish Universities Physics Alliance and STFC.

APPENDIX A: $f_{\pi}, f_{K}$ AND $f_{\eta_{s}}$

In [1, we analyzed simulation results for pion and kaon masses and decay constants obtained using the HISQ action for the valence quarks, with gluon configurations from MILC, produced using the ASQTAD action for the $\left(n_{f}=3\right)$ light sea quarks. We described how to extrapolate these results to the correct light-quark masses and to zero lattice spacing, obtaining decay constants that agree well with experiment.

Here we reuse our earlier simulation results, which are summarized in Table VIII (and in Table VI for the $\eta_{s}$ ), to extract a value for the static-quark potential parameter $r_{1}$. More importantly, we also extract from this analysis continuum values for the mass and decay constant

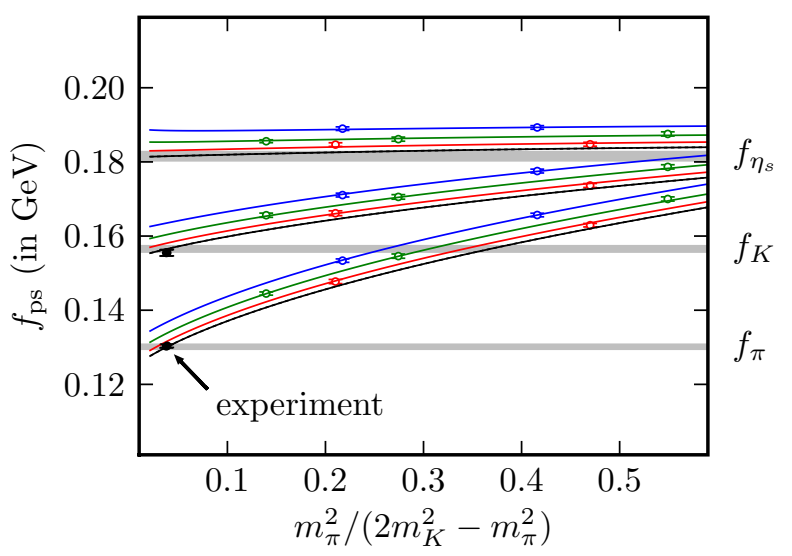

FIG. 4: The pseudoscalar decay constants plotted versus quark mass; $m_{\pi}^{2} /\left(2 m_{K}^{2}-m_{\pi}^{2}\right)$ is approximately the ratio of the $u / d$ to $s$ quark masses: $m_{l} / m_{s}$. The fit data is from lattice simulations with three different lattice spacings; results decrease with decreasing lattice spacing. The data have been adjusted to correspond to points where the sea-quark masses correspond to the valence masses. The lines are from the tuned fit function for each of the three lattice spacings. The bottom line in each group is the extrapolation to $a=0$. The gray bands indicate final values from the fit for the physical decay constants for all three mesons; the leftmost data points for $f_{\pi}$ and $f_{K}$ are the current experimental values.

TABLE VIII: Simulation results for pseudoscalar meson decay constants and masses (in lattice units) for several different lattice parameter sets (see Table $\Pi$ ),$u / d$ valence-quark masses $m_{l}$, and $s$ valence-quark masses $m_{s}$.

\begin{tabular}{cllcccc}
\hline \hline Set & $a m_{l}$ & $a m_{s}$ & $a f_{\pi}$ & $a m_{\pi}$ & $a f_{K}$ & $a m_{K}$ \\
\hline 1 & 0.0132 & 0.066 & $0.1152(3)$ & $0.2408(6)$ & $0.1290(4)$ & $0.4081(6)$ \\
2 & 0.0264 & 0.066 & $0.1254(4)$ & $0.3348(6)$ & $0.1345(4)$ & $0.4399(7)$ \\
\hline 3 & 0.0067 & 0.0537 & $0.0889(3)$ & $0.1567(4)$ & $0.1020(3)$ & $0.3242(5)$ \\
4 & 0.01365 & 0.05465 & $0.0957(4)$ & $0.2222(5)$ & $0.1060(4)$ & $0.3463(6)$ \\
5 & 0.0278 & 0.0525 & $0.1041(3)$ & $0.3113(6)$ & $0.1095(4)$ & $0.3727(6)$ \\
\hline 6 & 0.00705 & 0.0366 & $0.0647(2)$ & $0.1377(4)$ & $0.0731(3)$ & $0.2375(4)$ \\
7 & 0.01635 & 0.03635 & $0.0710(2)$ & $0.2050(4)$ & $0.0759(2)$ & $0.2594(4)$ \\
\hline \hline
\end{tabular}

of the $\eta_{s}$ meson. The masses and decay constants in Table VIII are obtained using the procedure described in Section II C for analyzing $\eta_{s}$ correlators; we treat all mesons the same way.

To extract a continuum value for $r_{1}$, we fit the decay constant data for lattice ensemble $i$ in Table VIII (and Table VI for $\left.a f_{\eta_{s}}\right)$ to

$$
a_{i} f_{\mathrm{ps}}\left(x_{a}, x_{b}, x_{l}^{\mathrm{sea}}, x_{s}^{\mathrm{sea}}, a_{i}\right)
$$

where $f_{\mathrm{ps}}$ is the formula from Appendix $\mathrm{B}$ and $(a, b)$ labels the valence quarks: $(l, l)$ for pions, $(l, s)$ for kaons and $(s, s)$ for $\eta_{s}$ s. The mass parameters $x_{a}, x_{b} \ldots$ are computed from the simulation masses in Table VIII. Parameter $r_{1}$ enters through the lattice spacing, which we 
take to be

$$
a_{i}=\frac{r_{1}}{\left(r_{1} / a\right)_{i}}
$$

where values for $\left(r_{1} / a\right)_{i}$ are given in Table I. We fit data from the pion, kaon and $\eta_{s}$ simultaneously since all of the fitting parameters are universal.

Our analysis here differs in three ways from our previous paper [1. First we are including the $\eta_{s}$ in our simultaneous analysis of the different pseudoscalar mesons; before we only included $\pi$ and $K$ mesons. Second we have re-expressed chiral perturbation theory in terms of pion and kaon masses rather than quark masses. This simplifies the analysis and also gives more reliable estimates for infrared quantities like chiral logarithms. We take the pion and kaon masses corresponding to the sea quark masses from [3] for ensemble sets $(3,4,6,7)$. Results for the other ensembles are not published so we generate approximate meson masses to go with the sea quarks by multiplying the meson masses for the valence quarks (Table VIII) by $\left(m^{\text {sea }} / m^{\text {val }}\right)^{1 / 2}$ (after converting HISQ quark masses into ASQTAD quark masses using Eq. (C1)). Replacing quark masses with meson masses in the chiral formulas gives results that agree well with our previous results.

The third difference from our earlier analysis is that here we require the fitting function to also fit experimental results for $f_{\pi}$ and $f_{K}$ at zero lattice spacing. We do this by treating the physical results as additional data to be fit, together with the simulation results, to a single parameterization. In our previous study we fit only simulation results, showing that these agreed with experimental data. Here our goal is different, as we seek an accurate value for $r_{1}$. That value is the one that allows the same chiral formulas to fit both our lattice results and the experimental results; $r_{1}$ is determined, in effect, from the experimental values for $f_{\pi}$ and $f_{K}$.

Our simulations omit both electromagnetic corrections and isospin-breaking effects. Following [22, we remove leading-order errors of both sorts by using

$$
\begin{aligned}
& m_{\hat{\pi}}^{2}=m_{\pi^{0}}^{2} \\
& m_{\hat{K}}^{2}=\frac{1}{2}\left(m_{K^{0}}^{2}+m_{K^{+}}^{2}-\left(1+\Delta_{E}\right)\left(m_{\pi^{+}}^{2}-m_{\pi^{0}}^{2}\right)\right)
\end{aligned}
$$

for the physical masses of the pion and kaon. $\Delta_{E}$ parameterises the violation of Dashen's Theorem which, in the chiral limit, states that the $K^{+}$and $\pi^{+}$have equal electromagnetic corrections, while the $\pi^{0}$ and $K^{0}$ have none. We take $\Delta_{E}=1(1)$. Electromagnetic corrections are also removed from the standard definition of the decay constants, whose values we take to be [15]:

$$
f_{\pi}=0.1304(5) \mathrm{GeV} \quad f_{K}=0.1555(9) \mathrm{GeV} .
$$

The fitting parameters that are varied in the fit include all of the parameters that define $f_{\mathrm{ps}}$ (see Appendix B, as well as $r_{1}$ itself. As discussed in Appendix B, all parameters have priors in our fits. For $r_{1}$ we take a very broad prior, $r_{1}=0.315(10) \mathrm{fm}$, that easily encompasses
TABLE IX: Extrapolation and other errors in our results from the chiral analysis of $\pi, K$, and $\eta_{s}$ masses and decay constants. Finite-volume errors are dealt with separately (see text).

\begin{tabular}{rllll}
\hline \hline & $r_{1}$ & $f_{\eta_{s}}$ & $m_{\eta_{s}}$ & $f_{\eta_{s}} / m_{\eta_{s}}$ \\
\hline$a^{2}$ extrapolation & $0.6 \%$ & $0.2 \%$ & $0.3 \%$ & $0.3 \%$ \\
$m_{q}$ extrapolations & 0.7 & 0.2 & 0.2 & 0.2 \\
$r_{1} / a$ uncertainty & 0.2 & 0.1 & 0.1 & 0.1 \\
initial uncertainty in $r_{1}$ & 0.6 & 0.1 & 0.0 & 0.1 \\
experimental errors in $\pi, K$ & 0.2 & 0.2 & 0.2 & 0.3 \\
statistical errors & 0.7 & 0.3 & 0.4 & 0.5 \\
\hline Total & $1.4 \%$ & $0.5 \%$ & $0.5 \%$ & $0.7 \%$ \\
\hline \hline
\end{tabular}

all current estimates; it has little impact on the final results.

The results of our fit are show in Figure 4. The fit is excellent, with a $\chi^{2}$ per degree of freedom of 0.4 . Our main results are physical (i.e., continuum) values for $r_{1}$ and for the decay constant and mass of the $\eta_{s}$ :

$$
\begin{aligned}
r_{1} & =0.3190(45)(20) \mathrm{fm}, \\
f_{\eta_{s}} & =0.1815(10)(2) \mathrm{GeV}, \\
m_{\eta_{s}} & =0.6858(38)(12) \mathrm{GeV} \\
f_{\eta_{s}} / m_{\eta_{s}} & =0.2647(18)(1)
\end{aligned}
$$

By "physical" we mean extrapolated to zero lattice spacing and the correct, physical values for the quark masses. We quote two errors here. The first is the fitting error, representing uncertainties from simulation statistics, and from the chiral and lattice-spacing extrapolation. A detailed breakdown of these errors is given in Table IX. The second error is equal to the size of the finite-volume correction. As discussed in Appendix B, finite-volume corrections are somewhat ambiguous for staggered-quark formalisms like HISQ. We choose to include finite-volume corrections, but, to be conservative, take half the size of the correction as an uncertainty.

We need the physical $\eta_{s}$ results for our analysis in Section IIC of the $\eta_{s}$ decay constant $f_{\eta_{s}}$. These fit results have statistical correlations with each other, as well as with the output value of $r_{1}$, the values of $r_{1} / a$ used in the fit, and the simulation results for $a f_{\eta_{s}}$ (Table VI). We used the fit here to compute means and a covariance matrix for all of these quantities, and this is used as input data in the $f_{\eta_{s}}$ analysis of Section IIC

Note that the values for the $\eta_{s}$ mass and decay constant agree to better than a percent with the leading-order expectations from chiral perturbation theory: $\left(2 m_{K}^{2}-\right.$ $\left.m_{\pi}^{2}\right)^{1 / 2}$ and $2 f_{K}-f_{\pi}$, respectively. Our analysis above, however, goes far beyond leading order (see Appendix B. Our $\eta_{s}$ results are also quite independent of the input prior for $r_{1}$; taking $0.3133(23) \mathrm{fm}$ as the prior, for example, causes shifts that are smaller than a quarter of a standard deviation. The $\eta_{s}$ parameters are most sensitive to the physical parameters for the pion and kaon. They can easily be corrected should there be small shifts 
in the values derived from experiment for $f_{\pi}$ and $f_{K}$. The changes in the $\eta_{s}$ parameters would be:

$$
\begin{aligned}
\Delta f_{\eta_{s}} & =0.6 \Delta f_{K}+0.2 \Delta f_{\pi} \\
\Delta m_{\eta_{s}} & =0.2 \Delta f_{K}-0.6 \Delta f_{\pi},
\end{aligned}
$$

where $\Delta f_{\pi}$ and $\Delta f_{K}$ are changes in the pion and kaon decay constants from the values used here.

\section{APPENDIX B: AUGMENTED CHIRAL FORMULAS}

We model light-quark pseudoscalar masses and decay constants using partially-quenched chiral perturbation theory, augmented with corrections for the finite lattice spacing. For simplicity we re-express chiral perturbation theory in terms of pion and kaon masses, in place of the quark masses, using

$$
\begin{aligned}
& x_{l}=\frac{m_{\pi}^{2} / 2}{\Lambda_{\chi}^{2}} \approx 0.007 \\
& x_{s}=\frac{m_{K}^{2}-m_{\pi}^{2} / 2}{\Lambda_{\chi}^{2}} \approx 0.17
\end{aligned}
$$

as expansion parameters, where

$$
\Lambda_{\chi} \equiv 4 \pi f_{\pi} / \sqrt{2} \approx 1.2 \mathrm{GeV} .
$$

We use the formulas through next-to-leading order from [20, together with higher-order corrections in $x_{l}$ and $x_{s}$ and finite- $a$ corrections. For example, we model the mass and lattice spacing dependence of the decay constants using

$$
f_{\mathrm{ps}}\left(x_{a}, x_{b}, x_{l}^{\mathrm{sea}}, x_{s}^{\mathrm{sea}}, a\right)=f^{\mathrm{NLO}}+\delta f_{\chi}+\delta f_{\text {lat }}
$$

where $f^{\mathrm{NLO}}$ is the chiral formula through next-to-leading order, $\delta f_{\chi}$ is the continuum correction due to higherorder mass corrections, $\delta f_{\text {lat }}$ is the correction due to the finite lattice spacing, and $(a, b)$ labels the valence quarks: $(l, l)$ for pions, $(l, s)$ for kaons, and $(s, s)$ for $\eta_{s} s$.

Our simulation results are not sufficiently accurate to resolve the difference between high-order polynomials in $x_{l}$ and $x_{s}$ and high-order logarithms, so we keep just the polynomials:

$$
\begin{aligned}
\delta f_{\chi} \equiv f_{0} & \left(c_{1}\left(x_{a}+x_{b}\right)^{2}+c_{2}\left(x_{a}-x_{b}\right)^{2}\right. \\
& +c_{3}\left(x_{a}+x_{b}\right)\left(2 x_{l}^{\text {sea }}+x_{s}^{\text {sea }}\right) \\
& +c_{4}\left(2 x_{l}^{\text {sea }}+x_{s}^{\text {sea }}\right)^{2}+c_{5}\left(2\left(x_{l}^{\text {sea }}\right)^{2}+\left(x_{s}^{\text {sea }}\right)^{2}\right) \\
& \left.+c_{6}\left(x_{a}+x_{b}\right)^{3}+c_{7}\left(x_{a}+x_{b}\right)\left(x_{a}-x_{b}\right)^{2}\right),
\end{aligned}
$$

where $f_{0}$ is the bare decay constant in chiral perturbation theory and the $c_{i}$ are expected to be $\mathcal{O}(1)$, except for seaquark terms where the coefficients should be $3-5$ times smaller. Still higher-order terms are smaller than $0.1 \%$ and so negligible, as are the last few terms in practice.
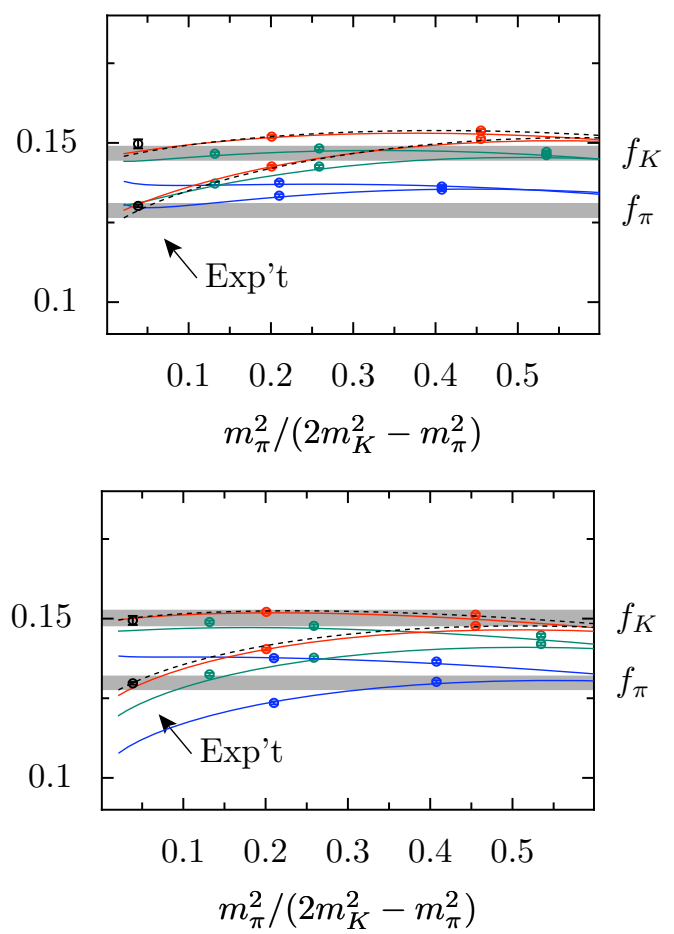

FIG. 5: Fits to two different sets of fake data for pion and kaon decay constants with very different $a^{2}$ behavior from each other and from the real simulation data (Figure 4). The "experimental" points indicated in each case correspond to the exact results, extracted from the formulas used to generate the fake data.

Following [1, we model the $a^{2}$ dependence using a mixture of terms that depend upon $a^{2}$ and $x_{l}$ :

$$
\begin{aligned}
\delta f_{\text {lat }}=f_{0} & \left(d_{1}\left(a \Lambda_{\mathrm{QCD}}\right)^{2} \alpha_{s}+d_{2}\left(a \Lambda_{\mathrm{QCD}}\right)^{2} \alpha_{s}^{3}\right. \\
& +d_{3}\left(a \Lambda_{\mathrm{QCD}}\right)^{2} \log \left(x_{l}\right) \alpha_{s}^{3} \\
& \left.+d_{4}\left(a \Lambda_{\mathrm{QCD}}\right)^{4}+d_{5}\left(a \Lambda_{\mathrm{QCD}}\right)^{5}\right)
\end{aligned}
$$

where we set $\alpha_{s}=\alpha_{V}(2 / a), \Lambda_{\mathrm{QCD}}^{2}=\left(4 m_{K}^{2}-m_{\pi}^{2}\right) / 3$ as in [20]. $\left(\Lambda_{\mathrm{QCD}}\right.$ is also the ultraviolet scale in the chiral logarithms.). We allow the coefficients to have mass dependence

$$
\begin{aligned}
d_{i} & =d_{i 1}+d_{i 2}\left(x_{a}+x_{b}\right) \\
& +d_{i 3}\left(2 x_{l}^{\text {sea }}+x_{s}^{\text {sea }}\right)+d_{i 4}\left(x_{a}^{2}+x_{b}^{2}\right) .
\end{aligned}
$$

Again the $d_{i j}$ are expected to be $\mathcal{O}(1)$, except for terms involving sea-quark terms which should be 3-5 times smaller. The highest-order terms in these expansions are already negligible, making further terms irrelevant. We include the $\log \left(x_{l}\right)$ term in Eq. (B6) to allow for nonanalytic behavior at small $x_{l}$, although in practice it is negligible in our fits.

We included priors in our fitting analysis for each of the parameters in $f^{\mathrm{NLO}}$ and for all the $c_{i} \mathrm{~S}$ and $d_{i j}$ s. These are initial estimates for each parameter that function as extra 
"data" and allow us to account (in our error estimates) for the uncertainties in these parameters, even when they are largely unconstrained by our simulation data. The parameters in $f^{\mathrm{NLO}}$ are well determined by our data; we use very broad priors for these, which have no impact on the final errors. We use a prior of $0(1)$ for each of the $c_{i} \mathrm{~s}$ and $d_{i j}$ s, except for terms involving sea-quark masses in which case we use $0.0(3)$.

As reported in [1, we have tested these fitting formulas extensively by using formulas from partially-quenched staggered chiral perturbation theory, with randomly selected coefficients and randomly generated higher-order corrections in the masses and $a^{2}$, to generate fake data sets for the same masses and lattice spacings used in our analysis here. We added statistical noise to the fake data that was comparable in magnitude to that in our real simulation data, with similar correlations. We then fit the fake data using the formulas above, together with the Empirical Bayes method [14] to set a prior for the expansion parameters $\left(c_{i}\right.$ and $\left.d_{i j}\right)$. In each case we could compare extrapolated results from our analysis of the fake data with the exact results, since we knew the underlying formula used to generate the fake data. We ran tests for several hundred cases. As expected, we found that $70 \%$ of the time the extrapolated results were within one standard deviation of the exact results. Two examples, shown in Fig. 5 , illustrate how effective our formulas are in handling $a^{2}$ dependence that is much larger and much more complex than we see in our actual simulation results (Figure 4),

The logarithms in the NLO chiral formulas reflect infrared sensitivity. These terms are sensitive to the finite volume of our lattice at the level of $0.1-1 \%$ for the decay constants (less for masses). We add finite-volume corrections to the logarithms which we obtain by recomputing the one-loop chiral corrections that lead to logarithms using finite-volume sums instead of integrals in momentum-space, and subtracting them from the infinite volume results. These corrections are quite sensitive to the meson mass, which raises an issue since in staggered-quark formalisms like HISQ each pseudoscalar meson comes in several different "tastes", all of them heavier than the Goldstone meson whose mass we use in our formulas. Taste splittings are $a^{2}$ corrections, which vanish in the continuum limit, and most of the effects of these we model with our corrections Eq. (B6) (which we have tested, as discussed in the previous paragraph). The finite-volume corrections, however, are particularly sensitive to meson masses, so we use an "effective" pseudoscalar mass when we calculate them:

$$
\left(m_{a b}^{\mathrm{eff}}\right)^{2}=\left(m_{a b}^{\mathrm{gs}}\right)^{2}+g_{m}\left(a / r_{1}\right)^{2}
$$

where $m_{a b}^{\mathrm{gs}}$ is the Goldstone meson's mass. We expect $g_{m} \approx 0.2 \mathrm{GeV}^{2}$. We allow $g_{m}$ to float in our fits, treating it as a fit parameter. We use $0.2(6)$ as our prior. Our fit favors a nonzero value for $g_{m}$, giving $g_{m}=0.2(3)$ which is consistent with expectations.

\section{APPENDIX C: SEA-QUARK MASSES}

We include terms in each of our fitting functions that correct for the discrepancies $\delta m_{q}^{\text {sea }}$ between the bare seaquark masses used in the simulation and the physically correct bare quark masses (that is, the ones that give correct masses for the $\pi, K$, and $\left.\eta_{s}\right)$. Our estimates for the correct $s$-quark masses (in lattice units) for each ensemble are given in Table VII the $u / d$ mass is 27.8(3) times smaller [1. These masses, however, are for HISQ quarks, while the sea quarks were all analyzed using the ASQTAD formalism. Quark masses in the two formalisms can be related to each other, ensemble by ensemble, by comparing $\pi$ and $\eta_{s}$ masses for mesons whose valence quarks are either HISQ or ASQTAD quarks. HISQ masses and ASQTAD masses are equivalent when they give the same $\pi$ and $\eta_{s}$ masses. The ratio of a HISQ mass to the corresponding ASQTAD mass determined in this way should be almost independent of the valence-quark mass, but will depend somewhat on the lattice spacing and weakly on the sea-quark masses. We have compared ASQTAD data from 3 for ensembles 3,4,6,7 with our results in Table VIII to obtain the following simplified parameterization for the ratio of HISQ to ASQTAD quark masses:

$$
\frac{a m^{\text {hisq }}}{a m^{\text {asq }}}=1.158 \frac{1+0.44\left(a / r_{1}\right)^{2}}{1+0.009\left(a m_{\text {tot }}^{\text {asq }} / a m_{s}^{\text {tuned }}\right)}
$$

where $m_{s}^{\text {tuned }}$ is the tuned HISQ mass given in Table VII and $m_{\text {tot }}^{\text {asq }}$ is the sum of the three sea-quark ASQTAD masses for that ensemble. This formula is accurate to a few percent.
[1] E. Follana et al, HOQCD collaboration, Phys. Rev. Lett, 100:062002 (2008) arXiv:0706.1726.

[2] C. T. H. Davies et al, HPQCD/Fermilab/MILC collaborations, Phys. Rev. Lett. 92:022001 (2004) arXiv:heplat/0304004.

[3] C. Aubin et al, MILC collaboration, Phys. Rev. D70:094505 (2004) [arXiv:0402030].

[4] R. Sommer, Nucl. Phys. B411, 839 (1994).

[5] A. Bazavov et al, arXiv:0903.3598.
[6] Taking the orbital excitation energy as the spin-averaged mass of $\chi(1 \mathrm{P})$ states minus the spin-average of vector and pseudoscalar (1S) states gives $457 \mathrm{MeV}$ from experiment for both $b \bar{b}$ and $c \bar{c}$. For the spin-average of $2 \mathrm{~S}$ minus $1 \mathrm{~S}$ states $c \bar{c}$ gives $606(1) \mathrm{MeV}$ and for $b \bar{b},(581-h / 4) \mathrm{MeV}$, where $h$ is the unknown hyperfine splitting at the $2 \mathrm{~S}$ level (of order 30-40 MeV) [15.

[7] A. Gray et al, HPQCD collaboration, Phys. Rev. D72:094507 (2005) arXiv:hep-lat/0507013. 
[8] B. A. Thacker and G. P. Lepage, Phys. Rev. D43, 196 (1991); G. P. Lepage et al, Phys. Rev. D46, 4052 (1992) arXiv:hep-lat/9205007.

[9] Note that there is a typographical error in the lattice Hamiltonian given in [7]. The Hamiltonian used there and in this work are identical.

[10] C. T. H. Davies et al, Phys. Rev. D50, 6963 (1994) arXiv:hep-lat/9406017.

[11] G. P. Lepage and P. Mackenzie, Phys. Rev. D48, 2250 (1993).

[12] Smearings suitable for ground-state hadrons are useful where there is a problem with expoonentially falling signal/noise, as in the $B$ system. This is not an issue for the $b \bar{b}$ system.

[13] E. Gregory et al, HPQCD collaboration, PoS(LATTICE 2008):109 arXiv:0810.1845.

[14] G. P. Lepage et al, Nucl. Phys. B (Proc. Suppl. 106), 12 (2002) arXiv:hep-lat/0110175.

[15] Particle Data Group, http://pdg.lbl.gov/
[16] C. Morningstar, Phys. Rev. D50, 5902 (1994) arXiv:heplat/9406002]; E. Mueller, Proceedings of LAT2009 arXiv:0909.5126.

[17] E. Follana et al, HPQCD collaboration, Phys. Rev. D75:054502 (2007) arXiv:hep-lat/0610092.

[18] The impact on our final value of $r_{1}$ from our $1.5 \%$ systematic error in $m_{D_{s}}-m_{\eta_{c}} / 2$ is reduced to $1.1 \%$ by the prior for the physical value of $r_{1}(=0.315(10) \mathrm{fm})$.

[19] S. Basak et al, MILC collaboration, PoS(LATTICE 2008):127 (2008).

[20] S. Sharpe and N. Shoresh, Phys. Rev. D62:094503 (2000) arXiv:hep-lat/0006017.

[21] Y. Aoki et al, arXiv:0903.4155.

[22] C. Aubin et al, MILC collaboration, Phys. Rev. D70:114501 (2004) arXiv:hep-lat/0407028.

[23] R. G. Edwards and B. Joo [SciDAC/LHPC/UKQCD Collaborations], Nucl. Phys. Proc. Suppl. 140832 (2005) arXiv:hep-lat/0409003. 